\title{
On An Argument Against Semantic Compositionality
}

\author{
Francis Jeffry Pelletier \\ Departments of Philosophy and Computing Science \\ University of Alberta \\ Edmonton, Alberta, Canada T6G 2E1 \\ jeffp@cs.ualberta.ca
}

\begin{abstract}
James Higginbotham (1986) presents a theory of semantic interpretation which violates the principle of semantic compositionality. He gives an argument by means of an example construction in favor of his contention. I show that compositioinal theories have more resources than some researchers give it credit for, and that these can be used in two different ways to account for the phenomenon Higginbotham describes.
\end{abstract}

\section{Introduction}

The Principle of Semantic Compositionality is the principle that the meaning of a complex expression is a function of (and only of) its parts together with the method by which those parts are combined. As stated, The Principle is vague or underspecified at a number of points, such as "what counts as a part," "what is a meaning,", "what kind of a function is allowed," and the like. But vagueness and underspecification hasn't stopped some people from treating it as an obviously true principle, true almost by definition; nor has it stopped some others from attacking it both on "empirical grounds" and on theoretico-methodological grounds. It seems to me that many of these discussions fail because of a lack of precision on just the previouslymentioned points, and that other discussions are best described as: "How compositionality can/can't be accommodated within theory X," rather than whether The Principle is or isn't true. In its most general form, for instance as stated above, The principle makes no assumptions about what the parts of a complex expression are, nor does it put any restrictions on what is the function on parts and mode of combination. Despite the fact that this is a very general way to state semantic compositionality, it is nonetheless possible to investigate certain claims concerning The Principle. For example, someone might claim that some linguistic phenomenon or other cannot be accommodated in accordance with The Principle. But so long as the person will commit him- or herself to some specified theory of meaning, and so longs the person will agree on what the range of relevant syntactic rules are, it becomes possible to try 
to give an account of the phenomenon which is in accordance with The principle. Of course, this alternative account may not satisfy the original person for one reason or another - for example the compositional account may not be "as elegant" or it may not be "systematically generalizable to other phenomena"; but the giving of such an account nonetheless shows that the original phenomenon is amenable to some compositional account. An it therefore brings to the forefront what is often hidden in these discussions: that the real disagreements are not really about matters of compositionality in the sense of The Principle, but are rather aesthetic judgements concerning "how well" the phenomenon is described.

\section{Higginbotham's Theory}

In this paper I wish to discuss an example, and a certain way of looking at semantics, which was put forward in Higginbotham (1986). Higginbotham, in that paper, wished to claim that any theory of natural language will be forced, ultimately, to give up The Principle. That is, he believed that no matter what syntactic level of description a theory uses (surface structure or some other level such as LF within a Government and Binding framework) to induce a "semantic reading", and no matter whether this "semantic reading" be a translation into an (arguably) compositional language like intensional logic or first-order quantification theory, or be "direct truth conditions" of either the model-theoretic or absolute sort, one will be forced to employ a noncompositional stage.

The sorts of theories of natural language which Higginbotham was concerned to argue against - Generalized Phrase Structure Grammars, Head-Driven Phrase Structure Grammars, Lexical-Functional Grammars, Montague Grammars, Categorical Grammars, and others - are compositional in the sense that they allow one to associate with any syntactic unit (syntactic unit in whatever sense the grammar defines, which will be different for these differing theories) a "logical form representation" for that unit. This representation is a function only of the representations for the syntactic units which make it up. Thus, for example, if a sentence $S$ is composed of a noun phrase NP and a verb phrase VP (only), then the ("logical form") representation of the $\mathrm{S}$ is a function only of the ("logical form") representations of the NP and the VP plus information about the relevant syntactic rule which combined the NP and the VP. If the VP is itself composed of two other VP's plus a connective, then the ("logical form") representation of this VP is a function only of the ("logical form") representations of the constituent VP's and of the connective, plus information about the relevant syntactic rule which combined with these VP's and connective. One never requires information about other parts of the sentence, but rather only about the subparts of the portion of the sentence that is currently being described.

Following Higginbotham, let us abstract away from the details of specific proposals for the choice of "semantic value" (that is, away from whether it is a model, a representation language, as sequence, etc.) and merely say

$$
\mathrm{V}(\mathrm{x}, \mathrm{B}, \mathrm{A})
$$


which is to be read: $x$ is the value of the phrase marker $B$ considered as a subphrase of phrase marker $A{ }^{1}$ As a help in appreciating the force of this view of semantics, consider the following schematic of a syntactic analysis:

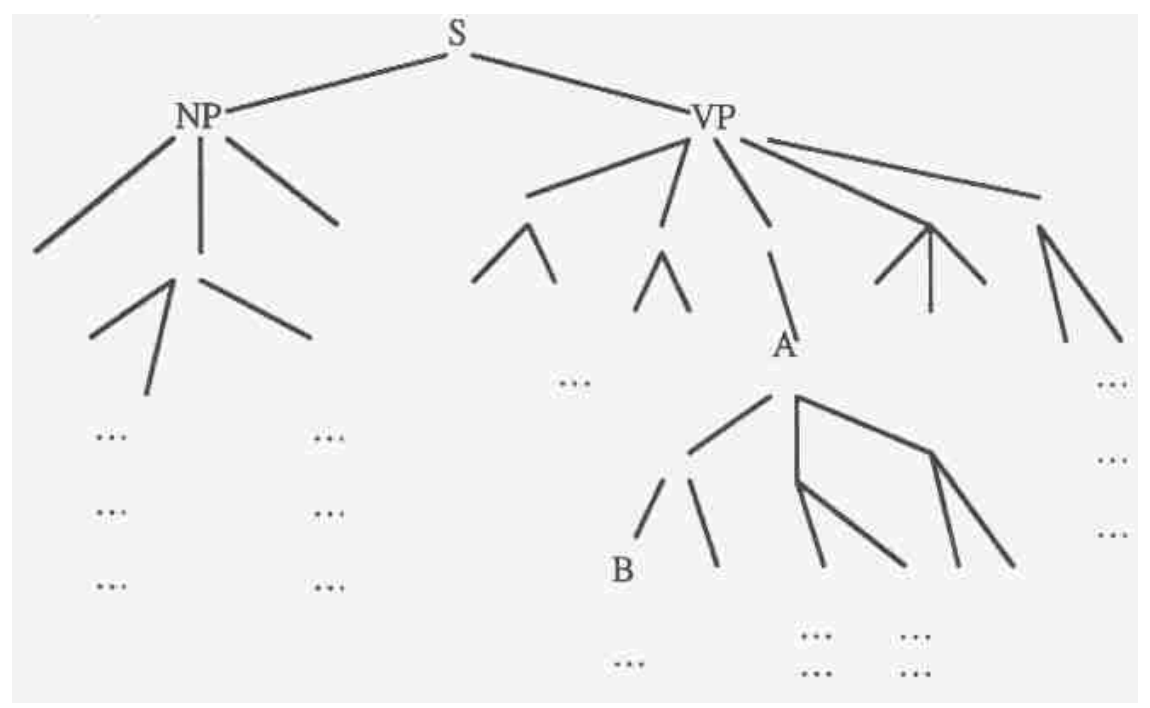

Higginbotham's idea is that the semantic value of node B might be one thing when it is considered as being a subnode of $\mathrm{A}$, but quite a different thing if it is considered as a subnode of VP, and yet another thing if it is considered as a subnode of $\mathrm{S}$. And in general, he thinks, it is the relation $\mathrm{V}(\mathrm{x}, \mathrm{B}, \mathrm{A})$ which we wish to define in general.

In some languages (artificial ones, in Higginbotham's opinion) the meaning of a node does not vary as function of which nodes dominate it in a syntactic analysis tree. These languages obey what Higginbotham calls The Indifference Principle:

$$
\mathrm{V}(\mathrm{x}, \mathrm{B}, \mathrm{A})=\mathrm{V}(\mathrm{x}, \mathrm{B}, \mathrm{B}) \text { for all } \mathrm{B} \text { and } \mathrm{A}
$$

That is, the semantic value of $\mathrm{B}$ never varies as a function of what dominates it. According to the Indifference Principle, a NP like all people is represented the same regardless of what larger syntactic context it occurs in: it would get the same representation in All people desire to learn as it does in John loves all people (assuming that the phrase all people is formed by the same syntactic rule in both cases). An in this

\footnotetext{
${ }^{1}$ Higginbotham has two further parameters for V: a "context parameter" and an assignmentof-values-to-variables parameter. Following Higginbotham, we will not consider these further parameters since they do not affect the point under consideration here. (But note that their presence is relevant to other discussions of compositionality - Higginbotham is making "context" be an element of the meaning of parts-and-wholes. This in turn raises further issues about what Compositionality amounts to in his theory. But we shall not pursue that further here.)
} 
last sentence, the meaning assigned to the NP node all people is fixed once and for all. It is not a different thing when on considers it in isolation from what it is when one considers it as a subnode of the VP nor again from what it is when one considers it as a subnode of the entire $S$. If in an (artificial?) language $\mathrm{V}(\mathrm{x}, \mathrm{B}, \mathrm{B})$ is itself determined by the semantic value of B's part only, then these languages obeying the Indifference Principle will also obey The Principle of Semantic Compositionality, and will be compositional in that pure and abstract sense. In fact, Higginbotham does believe that, even in natural languages, $\mathrm{V}(\mathrm{x}, \mathrm{B}, \mathrm{B})$ is entirely determined by the semantic values of B's parts only. ${ }^{2}$ It follows then that his only reason for denying The Principle of Semantic Compositionality is that he believes the Indifference Principle to be false.

\section{An Example}

Higginbotham presents an example which is designed to show the falsity of the Indifference Principle, and hence the falsity of The Principle of Semantic Compositionality. Keeping in mind the very general nature of Higginbotham's semantic proposals, we are not to take this example as showing only that some particular linguistic theory is committed to denying the Indifference Principle, but rather that any theory which can be described in the very general terms presupposed by viewing semantics as committed to the primacy of $\mathrm{V}(\mathrm{x}, \mathrm{B}, \mathrm{A})$ will have to deny the Indifference Principle.

Since this framework is so general, it amounts to including any theory at all that is committed to some semantic component or another. Thus the consequences of the example proposed by Higginbotham are serious indeed, and it behooves us to consider the true extent of its effects.

On the intuitive level, and independently of any theoretical framework, Higginbotham thinks that the unless in sentences like

(1) a. John will eat steak unless he eats lobster.

b. Every person will eat steak unless he eats lobster.

are to be represented with a disjunction - with an ' '. Using generalized quantifier notation, the (1) sentences would be represented as

(1') a. <John:x> (x eats steak $x$ eats lobster)

b. $<$ Every $x$ : person $(x)>(x$ eats steak $x$ eats lobster $)$

But a sentence like

(2) No person will eat steak unless he eats lobster.

\footnotetext{
${ }^{2}$ Because he has the other aspects of meaning - context and the like - already being a part of the meanings of the parts of B. See the discussion in footnote 1 above.
} 
does not use the unless as a disjunction. Instead, it is to be represented a

(2') <No x: person $(\mathrm{x})>$ (x eats steak \& $\square \mathrm{x}$ eats lobster).

From this evidence Higginbotham draws the conclusion that unless is to be represented as ' ' in some contexts and as ' $\& \square$ ' in other contexts. ${ }^{3}$ And from this conclusion he draws the moral that the Indifference Principle is false. How does he draw this further moral? Let's look at the details. According to Higginbotham, the syntactic structure of (2) is

(3):

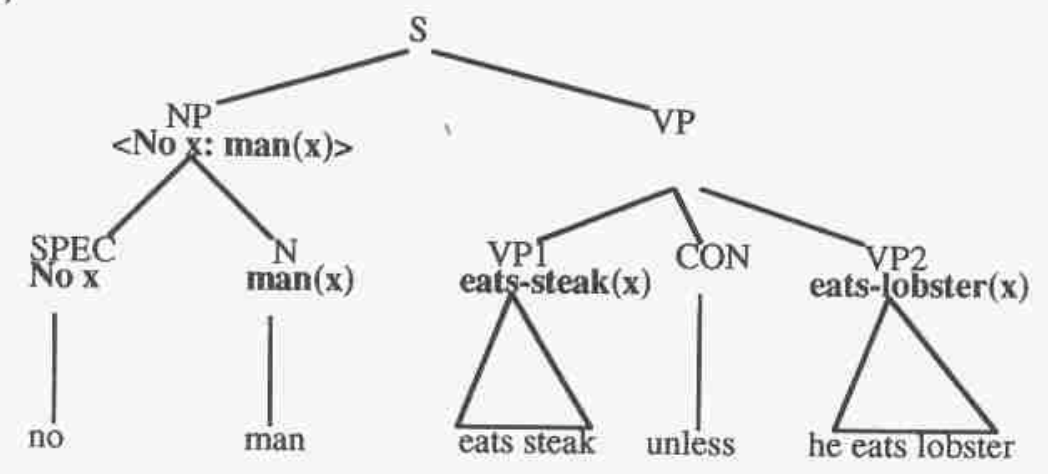

The semantic representations of the various nodes that can be computed are indicated in boldface. We should particularly note that we cannot compute the semantic value of the sentence $S$ until we know the value of VP, and that we cannot compute the

3 One might instead draw the conclusion that there is something amiss with generalized quantifiers, and that it is not the fault of unless violating the Indifference Principle. But it seems not. At least in so far as I understand generalized quantifiers, they obey these rules (PN is "proper name" and slash notation means "substitution for"):

$<\mathrm{PN}: \mathrm{x}>\square \mathrm{x} \square \square(\mathrm{PN} / \mathrm{x})$

$<$ Every $\mathrm{x}: \square \mathrm{x}>\square \mathrm{x} \square(\square \mathrm{x})(\square \mathrm{x} \square \square \mathrm{x})$

$<$ Some $\mathrm{x}: \square \mathrm{x}>\square \mathrm{x} \square(\square \mathrm{x})(\square \& \square \mathrm{x})$

$<$ No $\mathrm{x}: \square \mathrm{x}>\square \mathrm{x} \square<$ Every $\mathrm{x}: \square \mathrm{x}>\square \square \mathrm{x} \square(\square \mathrm{x})(\square \mathrm{x} \square \square \square \mathrm{x})$

And we get the correct representations, even with a No quantifier:

No person eats fish $\square<$ No $x$ : person $(x)>$ eats-fish $(\mathrm{x}) \square$

$(\square x)(\operatorname{person}(x) \square \square$ eats-fish $(x))$

We also get the right results even when the matrix sentence is a compound - even when it is a compound with or:

No person eats either steak or lobster $\square$

$<$ No $\mathrm{x}$ : person $(\mathrm{x})>($ eats-steak $(\mathrm{x}) \quad$ eats-lobster $(\mathrm{x}))$

$(\square \mathrm{x})(\operatorname{person}(\mathrm{x}) \square \quad($ eats-steak $(\mathrm{x}) \quad$ eats-lobster $(\mathrm{x})))$

So it looks like the generalized quantifier account handles the relevant phenomena correctly, and therefore the fault must lie with unless. 
value of VP until we know the value of CON. But recall that the value of $\mathrm{CON}$ is not fixed; it depends upon what sort of subject NP the sentence has. If that subject NP were John or were Every man, then the value of CON is ' '; but here the NP is No man and so the value of CON is ' $\& \square$ '. This means that, in order to compute the semantic value of some node B of the parse tree, we might, as here, need information from parts of the parse tree other than just what is dominated by B.

In the present case, we could unilaterally just decide to assign the lexical meaning of unless to be ' ' (or '\& $\square$ ' for that matter). But this will not help us anyway; for although we now could give the CON node a meaning (either ' ' or ' $\& \square$ ', whatever we decided), we still cannot give the correct meaning both to the VP node here for sentence (2) and also to the corresponding VP node for the sentences in (1). Whichever meaning we choose for unless will be fixed, and one or the other of these types of sentences will get the wrong VP meaning. The problem really is that we cannot tell which one to use without looking to the subject NP - but that node is not dominated by the VP node. Hence the Indifference Principle is false; for, although we might be able to compute $\mathrm{V}(\mathrm{x}, \mathrm{VP}, \mathrm{VP})$ as (eats-steak(x) eats-lobster(x)) (choosing as our meaning of unless), still, in order to compute $\mathrm{V}(\mathrm{x}, \mathrm{VP}, \mathrm{S})$ we need further information about other components of S. And if the Indifference Principle is false, then so is The Principle of Semantic Compositionality.

\section{Compositional Accounts of the Phenomenon}

I wish now to turn to an examination of the above argumentation that Higginbotham has used. I intend to show that compositional semantic theories have resources with which to meet this challenge, and that these resources stem precisely from the vagueness or lack of precision with which The Principle was initially stated. Thus in order to overturn The Principle, one will have to make it more precise than it is currently stated; but as soon as one does that, he is open to the charge of favoring one form of compositionality over some other form of compositionality...and that such favoritism amounts to merely an aesthetic choice with no scientific, theoretical, methodological, or logical underpinning. So I undertake this defense of The Principle not because I think that Semantic Compositionality is correct; indeed, I think that there are various severe methodological challenges one can mount against it. Rather, I merely wish to demonstrate the available resources, thereby indirectly indicating what sorts of future argumentation against The Principle will stand a chance of being successful.

One direction of argumentation against Higginbotham I will not pursue is to quibble over the syntactic representation of sentence (2). In fact I do not think that the above parse tree is correct; I believe that unless in such uses is a sentential connective and not a VP-connective. But such considerations would take us far afield from the arguments concerning Semantic Compositionality, which are what I want to consider here. I also will not carp at Higginbotham's suggestion that one meaning of unless is 
' $\& \square$ '. It is true that ' $\&$ ' is a binary connective and that ' $\square$ ' is a unary connective and that ' $\& \neg$ ' in this use does not make much sense. But I take Higginbotham's point to be that this meaning of unless should be represented as that binary connective whose truth conditions are given by saying that it is true when and only when the first argument is true and the second argument is false. And this understanding of Higginbotham's position makes perfectly good logic sense. (Whether it also accounts for the data remains to be seen.)

\subsection{Compositional Solution \#1: The Semantic Solution}

Compositional solution \#1 claims that unless is "vague". Its meaning is not " , (also not ' $\& \square$ ') but rather is merely "some connective or other." 4 Let's use '•' As a representation of this (vague) meaning, and we allow ourselves to $\square$-abstract over this meaning (we will use '•’ also as a variable over such meanings of connectives). We could then generate this representation

(4)

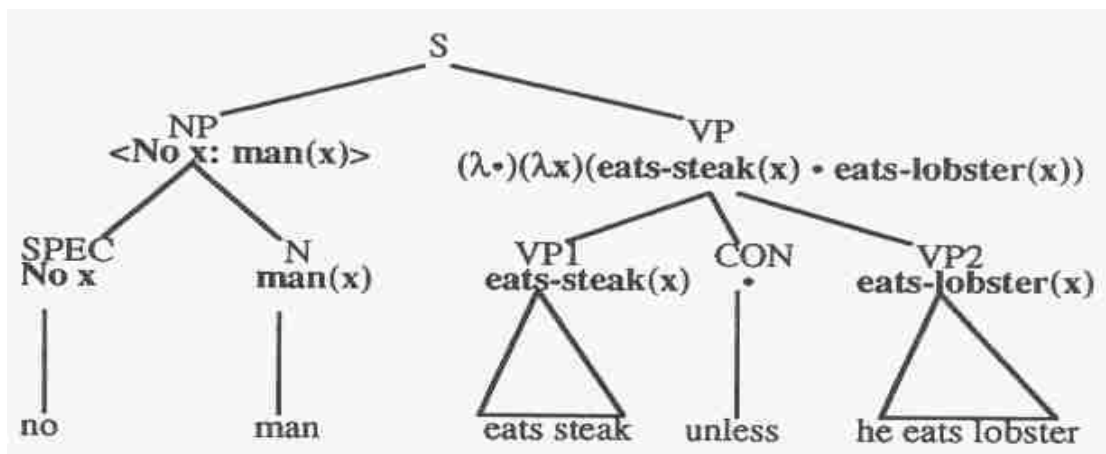

The value of VP here was computed in accordance with the Indifference Principle:

$$
\mathrm{V}(\mathrm{y}, \mathrm{VP}, \mathrm{VP})=(\square \bullet)(\square \mathrm{x})(\mathrm{VP1} \square \cdot \mathrm{VP} 2 \square
$$

where VP1 $\square$ and VP2 $\square$ are the meanings of the VP1 and VP2 syntactic parts. All this is done strictly with respect to the meanings of the immediate syntactic constituent parts and their mode of syntactic combination. This very same meaning of the VP would be generated no matter what the subject NP was: if the subject NP were John or were Every man we would nonetheless generate the displayed meaning for this VP.

We are now ready to compute the representation of S. Can we do this in accordance with the Indifference Principle for both the sentences in (1) and for (2)? The meaning of the Ss can be sensitive only to the representations of their NPs and VPs, and to the syntactic rule by which the NPs and VPs formed the Ss. So we must either decree that the sentences in (1) use a different syntactic rule than sentence (2), or we

4 Or better: "some connective or other from amongst this set ...". 
must find some differences in either the NPs or VPs. Although possible, it seems implausible to claim that the rule which combines NPs and VPs into Ss is a different one for the sentences in (1) than the rule used in (2). So any difference must come from the representations of the NPs or VPs. But this first compositional solution assumes that the representations for the VPs are the same, and therefore it must find the difference to reside in the meanings of the NPs.

And so it does; it supposes that the lexical meaning of no (as a quantifier) has associated with it a semantic feature of "negativity" and that this feature is preserved when no is combined into a quantified NP. All this is in accord with the Indifference Principle. Thus, according to this first compositional analysis, the semantic representation of the subject NP of the sentence has a (semantic) feature of "negativity". To complete the story, this analysis proposes that when computing the value of such sentences, one does function application of the VP-meaning to the NP-meaning, and (in the presence of the semantic "negativity" feature) $\square$-convert the $(\square \bullet)$ term to ' $\& \square$ ', thus yielding Higginbotham's desired sentence representation. Were we to apply the very same rule to one of the sentences in (1), we would find that the subject NP did not have this semantic "negativity" feature, and therefore when it came time to do the $\square$-conversion, the $(\square \bullet)$ term would become ' ${ }^{\prime}$. This is all done in accord with the Indifference Principle: no semantic rule depends on any information other than the semantic representations of the immediately dominated nodes together with the syntactic method of combination. Whatever one might think about the plausibility or generality of this sort of solution, the fact remains that it is in accord with the Indifference Principle and it shows that there is some analysis of the unless data which obeys Semantic Compositionality. Any other objections to the solution therefore must be "aesthetic".

\subsection{Compositional Solution \#2: The Syntactic Solution}

Compositional solution \#2 makes unless be "ambiguous" or better put, be two separate words. One of the words has the meaning ' ' and the other word has the meaning ' $\& \square$ '. The analogy is with such terms as slept, which is in fact two separate words, one of which has a meaning containing the semantic feature $[+\mathbf{+ s i n g}]$ and the other of which contains $[+\mathbf{p l}]$. The point is that somehow there are syntactic features which syntactically distinguish the two different words (in addition to whatever semantic differences there may be in their meanings). The grammar of English has a feature agreement rule which dictates that the subject NP must agree in this feature of plurality with the main VP. *Kim run and *Cheetahs runs are ruled out from the ranks of syntactically well-formed sentences because of this feature mismatch. For similar reasons

\section{(5) a. *Kim slept $[+\mathrm{pl}]$}

\footnotetext{
${ }^{5}$ Certain new tricks will need to be performed here to make $\square$-abstraction and $\square$-conversion mesh well with generalized quantifiers and with abstraction over connectives. There is no real mystery here, but the details would take us too far afield to be worth describing.
} 


\section{b. *The cheetahs slept [+sing]}

are ruled out from the set of well-formed sentences, as they violate the feature agreement rule. And this is despite the fact that the graphically (and aurally) identical sentences

a. Kim slept[+sing]

b. The cheetahs slept $[+\mathrm{pl}]$

are syntactically well-formed. If one thinks that the meaning of singular verbs is different from that of the corresponding plural verb (related, perhaps, in some such way as "how many activities are going on"), then when you encounter slept in a sentence, you have to bear in mind two different possible representations. Were one, in a bottom-up manner, to be (mechanically) parsing a sentence that contained slept, a choice would have to be made so as to be able to return to it should the choice turn out incorrect, or else the program could attempt to construct both parses simultaneously and discard the one which fails. These failures are recognized when there is a feature mismatch somewhere - e.g., when attempting to combine a subject NP with a main VP. And it is some such method as this which would syntactically distinguish the sentences in (6) from the non-sentences in (5).

In the case under discussion, we would have syntactic features [+neg] and [-neg]. And in our lexicon we would have that unless [-neg] means ' ' whereas unless [+neg] means ' $\& \square$ '. Not only is $[ \pm n e g]$ a feature of (some) connectives, but also it is a feature of certan NPs...it is inherited to the NP node at least from certain quantifiers that are marked as either $[ \pm n e g]$ in the lexicon. ${ }^{6}$ Thus no plus an $\mathrm{N}$ will make the [+neg] feature be inherited by the NP. The quantifier every will be marked [-neg] in the lexicon, and the rule that forms an NP from every plus an N will make the [-neg] feature be inherited by the NP. In this manner the sentences

a. *Every person will eat steak unless $[+$ neg] he eats lobster.

b. *No person will eat steak unless [-neg] he eats lobster.

will be marked as syntactically ill-formed. On the other hand, the graphically (and aurally identical sentences

a. Every person will eat steak unless [-neg] he eats lobster.

b. No person will eat steak unless $[+$ neg] he eats lobster.

will be counted as syntactically well-formed. And since each of the two unless's has the meaning appropriate to generate the desired VP meaning for those particular sentences, the proposal has the effect of generating the sentence meaning that Higginbotham desires both the sentences in (1) and for those in (2). And this generation is

6 There may also be other ways for such features to be introduced by the phrase structure rules, but that is not of concern to us here. 
always done in accordance with the Indifference Principle. Therefore The Principle of Semantic Compositionality is upheld.

\section{Conclusion: The Two Solutions}

The differences between the two compositional solutions to Higginbotham's puzzle are: (a) Solution \#1 gives one meaning to the VP...which is "vague" and which is "particularized" correctly by the subject NP, whereas Solution \#2 gives two possible meanings to the VP....and requires the subject NP to select one of the meanings correctly. Rather than treating unless as "vague" (as solution \#1 does), it instead treats it as "ambiguous". (b) Solution \#1 uses a semantic property of "negativity" to "particularize" the VP meaning, whereas Solution \#2 uses a syntactic property of "negativity" to enforce a feature co-occurrence restriction.

Solution \#1 illustrates the strategy of taking advantage of The Principle's underspecification of what "meaning of the parts" amounts to. It shows that it is possible to overcome the apparent violation of functionality by postulating a single "vague" or "more general" meaning. Solution \#2 illustrates the strategy of taking The Principle's underspecification of "what counts as parts." It shows the possibility of overcoming the apparent violation of functionality by postulating two distinct sets of parts. These two strategies are always open to one who wants to maintain Semantic Compositionality. The issue with either approach then becomes one of determining whether the postulation of "peculiar parts" or of "peculiar meanings-of-parts" makes the overall theory unacceptable on the other grounds. But as I said before, that is a different matter from whether there is a compositional solution to the phenomenon at hand. $^{7}$

\footnotetext{
7 My thanks to the members of (now sadly deceased) University of Alberta Logical Grammar Study Group for many helpful comments on this topic: Matthew Dryer (now at SUNY Buffalo), Fernanda Ferreira (now at Michigan State Univ.), Brendan Gillon (now at McGill Univ.), Bernard Linsky (still at Univ. Alberta!), Len Schubert (now at Univ. Rochester). I also wish to thank David Braun, Greg Carlson, and Sandro Zucchi for discussions on the general topic of semantic compositionality. This research was supported in part by the Canadian NSERC grant OPG5525. The author is a member of the Institute for Robotics and Intelligent Systems and wishes to acknowledege the support of the Networks of Centres of Excellence Program of the Government of Canada, NSERC, and the participation of PRECARN Associates Inc.
} 


\section{References}

Higginbotham, James (1986) "Linguistic Theory and Davidson's Program in Semantics: in E. LePore (ed.) The Philosophy of Donald Davidson: Perspectives on Truth and Interpretation (Oxford: Blackwell's). 